\title{
Prótese parcial fixa conservadora com duplo sistema de fixação: relato de caso
}

\author{
Conservative fixed partial denture with double fixation system: \\ case report
}

Wilson Matsumoto*

Rossana Pereira de Almeida*

Takami Hirono Hotta**

\section{Resumo}

Objetivo: apresentar uma alternativa conservadora de tratamento, com duplo sistema de fixação, em um caso de insucesso com prótese parcial fixa adesiva. Relato de caso: paciente do gênero feminino, 41 anos de idade, compareceu para tratamento na Faculdade de Odontologia de Ribeirão Preto com uma prótese parcial fixa adesiva, na região do dente 36, com deficiência na retenção e necessidade recorrente de fixação. No exame clínico, verificou-se desadaptação dos retentores metálicos nas faces linguais dos dentes pilares, tornando inviável a refixação da peça. Os preparos realizados nos dentes apresentavam deficiências na extensão e na ausência de apoios nas cristas marginais e paralelismo entre os dentes preparados. Como tratamento, foram realizadas restauração da morfologia dos dentes pilares com resina composta e confecção de uma prótese parcial fixa ultraconservadora, que resultou na manutenção das características de preparos com reduzido desgaste, restabelecimento da estética e da oclusão e eficácia na retenção da peça protética. Considerações finais: pode-se considerar que a prótese parcial fixa ultraconservadora, além de indicada para pequenos espaços edêntulos, com objetivo principal de preservação de estrutura dental, apresenta excelente sistema de fixação no substrato e pode ser utilizada como terapia alternativa em caso de insucesso da prótese parcial fixa adesiva.

Palavras-chave: Prótese parcial fixa. Resina composta. Cerâmica.

\section{Introdução}

O planejamento para reposição protética de pequenos espaços desdentados pode ser um problema para o profissional, principalmente, quando os dentes adjacentes são íntegros ${ }^{1}$, e, apesar de os implantes dentários serem uma ótima opção de tratamento ${ }^{2}$ para reposição de um elemento dental ausente, em algumas situações decorrentes da condição de saúde sistêmica prejudicada ou mesmo por opção do paciente, o tratamento de escolha pode ser uma prótese parcial fixa.

No entanto, além dos transtornos funcionais, estéticos, emocionais e sociais que a perda do elemento dental pode ocasionar ao indivíduo ${ }^{3}$, deve ser explicado que, optando por uma prótese fixa convencional, haverá a necessidade de desgaste de dentes pilares, que, muitas vezes, podem estar íntegros. E, visto que a reposição protética deve não só considerar esses aspectos, como também ser realizada com o mínimo de prejuízo biológico ao paciente ${ }^{4}$, opções alternativas de tratamento devem ser propostas.

A prótese parcial fixa adesiva (PPFA) é um tipo de prótese que apresenta a característica de reversibilidade, pois requer preparos minimamente invasivos, que não chegam a comprometer os dentes pilares ${ }^{5}$. Embora os preparos sejam mínimos, existe o desgaste de esmalte dental, que elimina a convexidade natural do dente nas faces proximais e lingual ou palatina.

O objetivo deste trabalho é apresentar uma alternativa conservadora de tratamento, com duplo sistema de fixação da peça, em um caso de insucesso com prótese parcial fixa adesiva.

\footnotetext{
Doutor em Reabilitação Oral, professor associado da área de Prótese Parcial Fixa, Departamento de Materiais Dentários e Prótese, Faculdade de Odontologia de Ribeirão Preto, Universidade de São Paulo.

Doutora em Reabilitação Oral, professora associada da área de Prótese Parcial Fixa, Departamento de Materiais Dentários e Prótese, Faculdade de Odontologia de Ribeirão Preto, Universidade de São Paulo.

*** Doutora em Reabilitação Oral, professora associada da área de Prótese Parcial Fixa, Departamento de Materiais Dentários e Prótese, Faculdade de Odontologia de Ribeirão Preto, Universidade de São Paulo.
} 


\section{Relato de caso}

O caso clínico foi submetido e aprovado pelo Comitê de Ética em Pesquisa da Faculdade de Odontologia de Ribeirão Preto, da Universidade de São Paulo, por meio do processo número 2014.1.1145.58.0.

Paciente do gênero feminino, 41 anos de idade, ausência do dente 36 reabilitado com uma PPFA com deficiência na retenção e necessidade recorrente de fixação compareceu para tratamento queixando-se da situação incômoda e relatando ter desistido de usar a prótese, mas desejando uma resolução rápida e eficiente.

No exame clínico, verificou-se desadaptação dos retentores metálicos nas faces linguais dos dentes pilares, tornando inviável a fixação da peça (Figura 1). Os preparos realizados nos dentes apresentavam deficiências nos limites de extensão, que deveriam proporcionar, por lingual, um abraçamento do dente, em torno de 180 graus $^{5}$. Verificou-se, também, ausência de preparos dos apoios nas cristas marginais e paralelismo entre os dentes preparados (Figura 2). Apesar disso, o caso poderia ter sido reconduzido para uma PPFA, no entanto, devido à resistência da paciente, optou-se pela prótese parcial fixa ultraconservadora (PPFU).

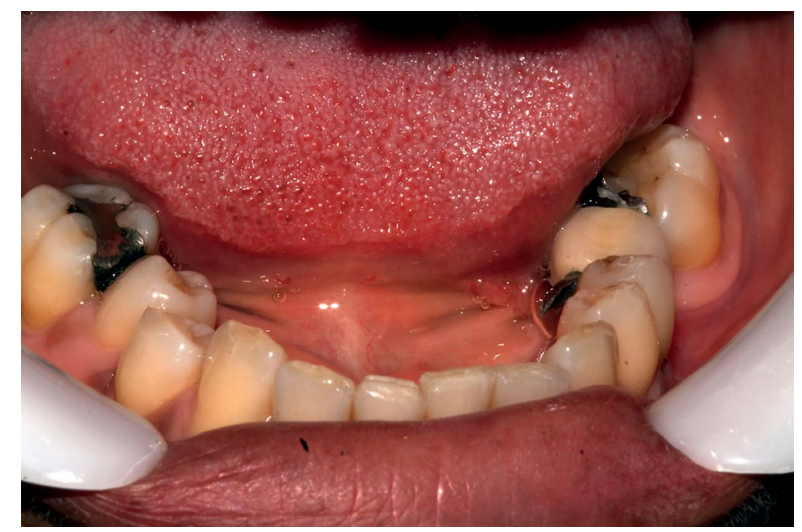

Figura 1 - Prótese parcial fixa adesiva desadaptada

Fonte: dos autores.

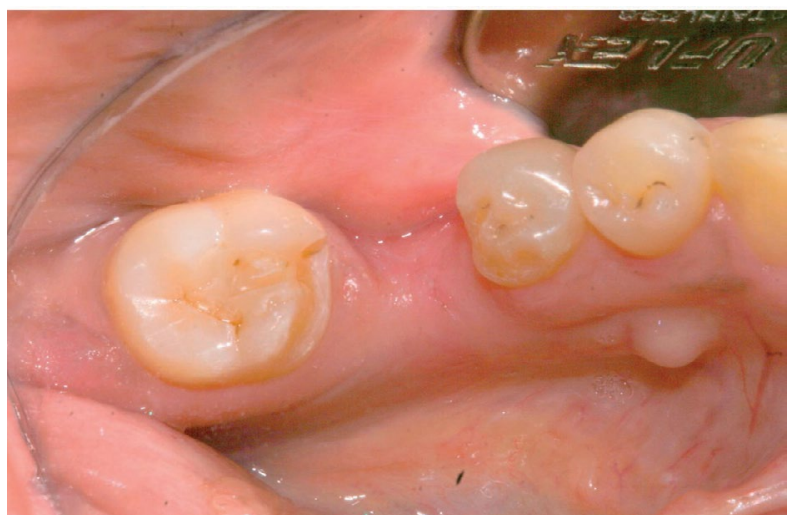

Figura 2 - Preparos existentes para a prótese parcial fixa adesiva Fonte: dos autores.

Inicialmente, os dentes pilares foram restaurados com resina composta (Z 350, 3M), para recompor a morfologia das superfícies. O preparo específico $0^{6}$ foi realizado nas faces oclusal e distal do dente 35 e nas faces oclusal e mesial do dente 37, com ponta diamantada tronco cônica ( $\mathrm{n}^{\circ}$. 2136, KG Sorensen), abrangendo um terço da dimensão vestíbulo-lingual do dente, seguindo o contorno dos istmos da superfície ${ }^{7}$, parede axial levemente divergente ${ }^{8}$, profundidade caixa oclusal em torno de $1,5 \mathrm{~mm}$ a $2 \mathrm{~mm}$ e uma depressão de $0.5 \mathrm{~mm}$ a $1 \mathrm{~mm}$ de profundidade no assoalho pulpar na área de encaixe da peça ${ }^{7}$, parede gengival da caixa proximal com $1 \mathrm{~mm}$ de profundidade, ao nível ou supra gengival ${ }^{9}$, e ausência de ângulos vivos ${ }^{10}$ (Figura 3).

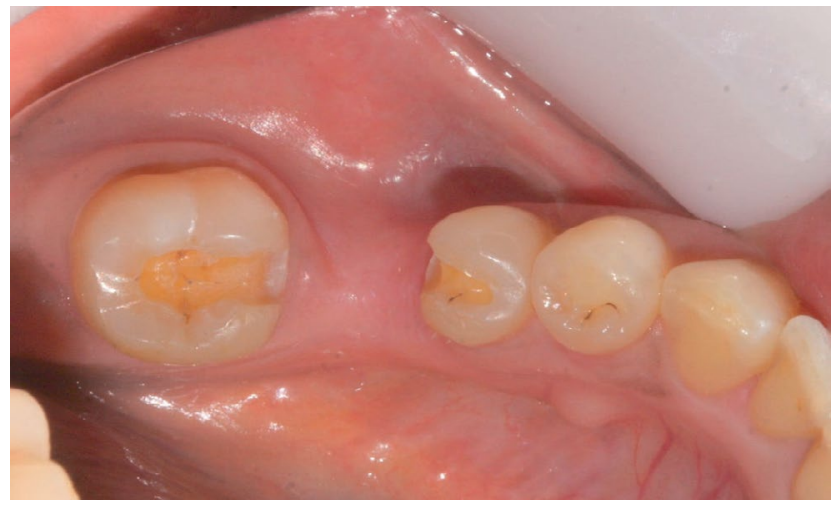

Figura 3 - Preparos realizados para a prótese parcial fixa ultraconservadora

Fonte: dos autores.

Após o término dos preparos, procedeu-se à moldagem com silicona de condensação (Zetaplus e Oranwash Zhermack, Italy). Restaurações provisórias foram feitas em resina acrílica (Dencor Classico - Brasil), ajustadas e fixadas com cimento provisório RelyX Temp NE (3M ESPE).

$\mathrm{Na}$ prova clínica, a adaptação da estrutura metálica é um procedimento relevante, similar à avaliação da adaptação da infraestrutura metálica de uma prótese fixa convencional, na qual a estrutura metálica deve estar perfeitamente adaptada na região de término gengival da caixa proximal, verificada por meio de visão direta e de instrumento clínico (Figura 4). Em casos de dificuldade de visão direta, tomadas radiográficas periapicais podem ser feitas.

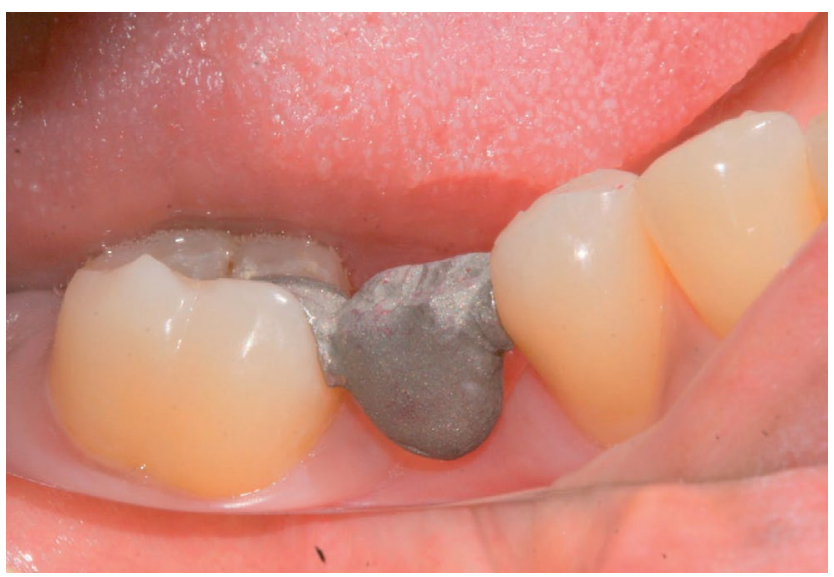

Figura 4 - Prova clínica da prótese parcial fixa ultraconservadora Fonte: dos autores. 
A aplicação da cerâmica é feita na região de pôntico, deixando os retentores apenas com opaco cerâmico aplicado. Na sequência clínica, a peça foi posicionada sobre os preparos e reavaliada quanto a forma, contorno, cor, adaptação e oclusão.

Previamente à cimentação, condicionou-se o opaco cerâmico dos retentores com ácido fluorídrico a 10\% (Condacporcelana, Dentsply Ltda., Brasil), por 1 minuto; após a lavagem com água e secagem, realizou-se aplicação de silano (Rely X Ceramic Primer, 3M ESPE). Os dentes pilares receberam profilaxia com pedra pomes e taça de borracha. Os procedimentos para fixação com cimento resinoso autoadesivo (RelyX-U200, 3M ESPE) seguiram as orientações do fabricante (Figura 5). Ainda com isolamento absoluto, foi realizado o condicionamento dos dentes pilares com ácido fosfórico a 37\% (Condac 37, FGM, Brasil), por 20 segundos, lavagem abundante com água, secagem dos dentes, aplicação de adesivo (Single Bond, 3M ESPE) e inserção de camadas de resina composta (Z350, 3M ESPE) nas cavidades e sobre os retentores, até o restabelecimento da morfologia e dos contatos oclusais (Figura 6).

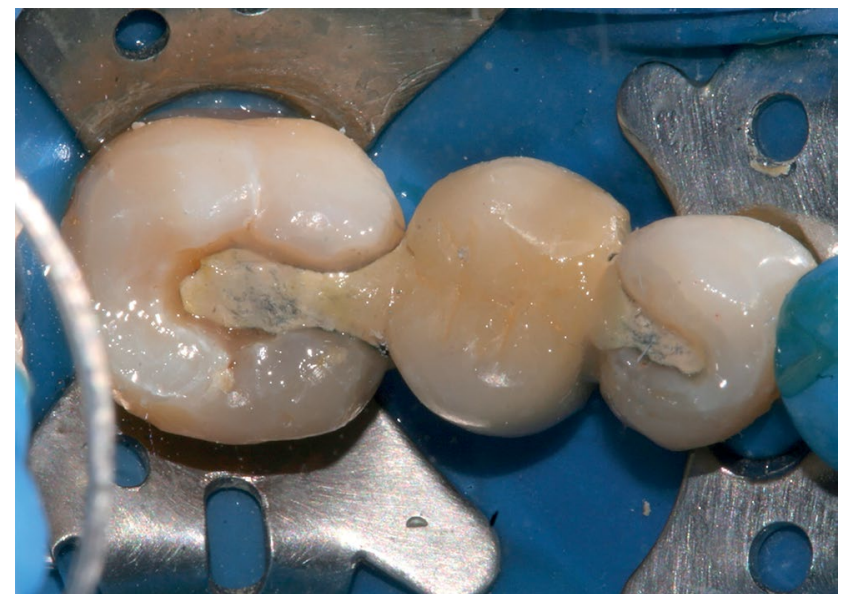

Figura 5 - Fixação da prótese parcial fixa ultraconservadora Fonte: dos autores.

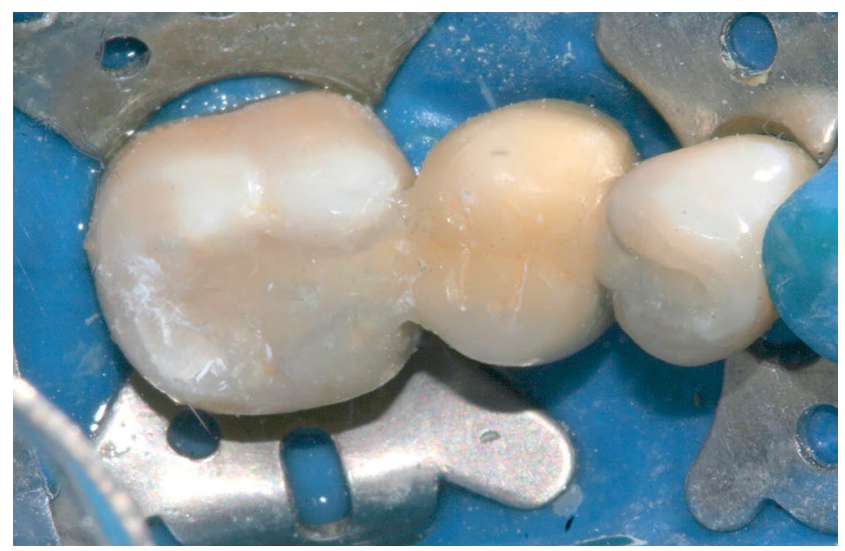

Figura 6 - Restaurações com resina composta Fonte: dos autores.

\section{Discussão}

A reposição protética de um elemento dental, quando o implante não é considerado uma opção, pode ser uma tarefa de difícil resolução, principalmente, quando os dentes pilares são hígidos ou com pequenas restaurações.

A escolha pelo tratamento com uma PPFA em detrimento de uma convencional considera a perspectiva clínica de preservação da estrutura dental pelo fato de os preparos serem menos invasivos ${ }^{4,9}$. Além disso, pelo não comprometimento da estrutura dos dentes pilares, há a característica de reversibilidade desse tipo de prótese ${ }^{5}$, na eventualidade de insucessos ou mudanças no planejamento.

Apesar das diversas vantagens da PPFA ${ }^{5,9,11}$, alguns estudos encontraram alta porcentagem de insucesso, após algum tempo de uso ${ }^{12,13}$, como necessidade de nova fixação da peça (recimentação), ou mesmo, impossibilidade de recimentação $0^{8}$, como ocorrido no presente caso.

Além de os preparos da PPFA serem, basicamente, em superfícies dentais lisas, devem ser realizados, principalmente, na camada de esmalte, e, por requererem limites de extensão amplos, áreas de difícil acesso podem estar envolvidas. Isso pode acarretar deficiências clínicas, que serão repassadas, por meio de modelos de trabalho imprecisos, ao laboratório de prótese, o qual irá confeccionar estruturas sobre superfícies não devidamente preparadas, aquém dos limites de extensão ou com sobre contornos, prejudicando o resultado final.

No presente caso, foram verificadas deficiências quanto aos limites de extensão $0^{5}$, ausência de apoios para travamento vertical da prótese, impedindo o deslocamento no sentido gengival ${ }^{1}$, e falta de paralelismo das paredes proximais ${ }^{8}$. Outros quesitos igualmente importantes, porém não passíveis de avaliação neste caso, são os cuidados na cimentação da peça, desde a seleção do agente de fixação até a execução do procedimento segundo as orientações do fabricante e o equilíbrio oclusal para minimizar cargas excessivas, evitando a fadiga e o estresse da peça e do agente de cimentação.

A escolha pela PPFU considerou a adequação de suas indicações com a condição clínica apresen$\operatorname{tada}^{6,7}$, ou seja, reabilitação de pequenos espaços, preservação de estrutura e da saúde do dente, paciente avesso ao preparo para coroa total ${ }^{7}$, presença de dentes pilares com restaurações oclusais ou ocluso-proximais e quando o implante dentário ${ }^{5}$ não for a opção selecionada ${ }^{2}$. Nesse caso clínico, além da reposição de um elemento dental, em substituição a uma PPFA, preservando suas características quanto a estética, conservação e funcionalidade, adicionalmente, teve a função de melhorar a capacidade de retenção, tendo em vista a utilização de retentores metálicos inseridos no interior das caixas proximais preparadas ${ }^{14}$. 
Para melhorar a adaptação, a inserção dos retentores metálicos nas caixas proximais e aumentar a forma de retenção e resistência, foram realizados ajustes em esmalte, nas faces proximais dos dentes, melhorando o paralelismo entre as paredes ${ }^{15}$. Esse procedimento poderia ter sido realizado no plano de tratamento com a PPFA e, potencialmente, colaborado com os aspectos biomecânicos como trajetória de inserção e adaptação.

Em toda reabilitação, é interessante que sejam analisadas as condições previamente existentes nos dentes pilares. Considerando o quesito de preservação de estrutura dental associada à necessidade de retenção da peça protética pela inserção dos retentores, neste trabalho, principalmente no dente 37 , os preparos foram realizados basicamente pela remoção de restaurações pré-existentes, e na inexistência delas, como no dente 35 , mantendo ao máximo as estruturas dentais sadias, sem a necessidade de tornar as paredes expulsivas.

Nas faces proximais, as margens dos preparos, estando situadas acima do nível da gengiva, permitiram que os procedimentos de moldagem fossem mais simples, sem necessidade de afastamento gengival, além de ser adequado do ponto de vista biológico e de higienização ${ }^{10}$.

Como a seleção do agente de cimentação deveria ser baseada nas necessidades específicas de cada situação clínica, optou-se por um cimento resinoso autoadesivo pelas suas propriedades e também para reduzir o número de procedimentos clínicos. Neste caso clínico, um fator relevante foi a característica da dupla fixação da peça protética aos dentes pilares. A fixação da PPFU ocorreu, inicialmente, pela ação do cimento inserido entre os retentores e os dentes preparados (parede pulpar e parede gengival), cumprindo sua principal função de preenchimento do vazio entre uma restauração protética e o dente, travando mecanicamente a restauração no local para evitar deslocamento durante a função $\mathrm{e}$, adicionalmente, pela técnica do condicionamento ácido do esmalte, aplicação do sistema adesivo e inserção da resina composta sobre os retentores e demais paredes dos preparos, restaurando os dentes pilares similarmente a uma restauração classe II com resina composta.

Além disso, a PPFU apresenta a característica de reversibilidade, ou seja, caso haja alteração de planejamento, e o indivíduo opte por implantes dentários, a prótese pode ser retirada e os dentes pilares restaurados com procedimentos de dentística restauradora.

\section{Considerações finais}

A prótese parcial fixa ultraconservadora, além de indicada para pequenos espaços edêntulos e com o objetivo principal de preservação de estrutura dental, pode ser utilizada como terapia alternativa em caso de insucesso da prótese parcial fixa adesiva, com a segurança da dupla fixação da peça.

\section{Abstract}

Objective: to present an alternative conservative treatment with a double fixation system for a case of failure with adhesive fixed partial denture. Case report: a 41 year-old female patient seeking treatment at the Dental School of Ribeirão Preto, SP, Brazil, with an adhesive fixed partial denture in the region of tooth 36 with retention deficiency and recurrent need for fixation. In the clinical examination, the metal retainers presented maladaptation on the lingual surfaces of the abutment teeth, rendering denture refixation ineffective. The preparations made on the abutment teeth presented deficiencies in the extension, absence of supports in the marginal ridges, and parallelism between the prepared teeth. The treatment choice included restoring the morphology of abutment teeth with composite resin and producing an ultra-conservative fixed partial denture, which preserved the characteristics of reduced-wear preparations, restored aesthetics and occlusion, and made prosthesis retention successful. Final considerations: it may be considered that the ultra-conservative fixed partial denture, besides being indicated for small edentulous spaces mainly for preserving tooth structure, presents an excellent fixation system on the dental substrate and may be used as an alternative therapy in case of failure of the adhesive fixed partial denture.

Keywords: Fixed partial denture. Composite resin. Ceramic.

\section{Referências}

1. Wyatt CCL. Resin-bonded fixed partial dentures: What's new? J Can Dent Assoc 2008; 73(10):933-8.

2. Stevenson RG, Refela JA. Conservative and esthetic cast gold fixed partial dentures- inlay, onlay, and partial veneer retainers, custom composite pontics, and stress-breakers: Part I: Fundamental design principles. J Esthet Restor Dent 2009; 21(6):365-74.

3. Hebling E, Pereira AC. Oral health-related quality of life: a critical appraisal of assessment tools used in elderly people. Gerodontology 2007; 24(3):151-61.

4. Edelhoff D, Sorensen JA. Tooth structure removal associated with various preparation designs for anterior teeth. $\mathrm{J}$ Prosthet Dent 2002; 87(5):503-9.

5. Durey KA, Nixon PJ, Robinson S, Chan MF. Resin bonded bridges: techniques for success. Brit Dent J 2011; 211(3):113-8.

6. Matsumoto W, Antunes RPA, Fernandes RM, Orsi EA, Hotta TH. Ultraconservative fixed partial denture: esthetic and preservation of dental structure. RGO Rev Gaúch Odontol 2014; 62(2):173-8.

7. Lacy A. The submerged framework bridge: laboratory and clinical considerations. Quint Dent Technol 2000; 23:139-48.

8. Aggstaller H, Beuer F, Edelhoff D, Rammelsberg P, Wolfgang Gernet W. Long-term clinical performance of resin-bonded fixed partial dentures with retentive preparation geometry in anterior and posterior areas. J Adhes Dent 2008; 10(4):301-6. 
9. Edelhoff D, Sorensen JA. Tooth structure removal associated with various preparation designs for posterior teeth. Int J Periodontics Restorative Dent 2002; 22(3):241-9.

10. Opdam NJ, Bronkhorst EM, Loomans BA, Huysmans MC. 12-year survival of composite vs. amalgam restorations. J Dent Res 2010; 89(10):1063-7.

11. Tredwin CJ, Setchell DJ, George GS, Weisbloom M. Resin-retained bridges as predictable and successful restorations. Alpha Omegan 2007; 100(2):89-96.

12. Hussey DL, Wilson NH. The provision of resin-bonded bridgework within the General Dental Services 1987-1997. Prim Dent Care 1999; 6(1):21-4.

13. Patsiatzi E, Grey NJ. An investigation of aspects of design of resin-bonded bridges in general dental practice and hospital services. Prim Dent Care 2004; 11(3):87-9.

14. Iglesia-Puig MA, Arellano-Cabornero A. Inlay fixed partial denture as a conservative approach for restoring posterior missing teeth: a clinical report. J Prosthet Dent 2003; 89(5):443-5.

15. Livaditis GJ. Cast metal resin-bonded retainers for posterior teeth. J Am Dent Assoc 1980; 101(6):926-9.

\section{Endereço para correspondência:}

Wilson Matsumoto

Faculdade de Odontologia de Ribeirão Preto -

Universidade de São Paulo

Avenida do Café, s/n, Campus USP

CEP 14040-904 Ribeirão Preto, São Paulo, Brasil

E-mail:wmatsumoto@forp.usp.br

Recebido: 01/07/2017. Aceito: 31/07/2017. 